\title{
Correlative Study Between the Sesamoid Bones under the Head of the First Metatarsal and the Development of Hallux Valgus Determined with Radiographs
}

Xiaozhong Li ( $\nabla$ lixiaozhong925@163.com )

Gansu University of Traditional Chinese Medicine

Dongxue Liu

The First Clinical Medical College of Gansu University of Chinese Medicine

Xufang Wang

The First Clinical Medical College of Gansu University of Chinese Medicine

\section{Research}

Keywords: Hallux valgus, Metatarsus, Sesamoid bones, Dislocation, X-rays

Posted Date: April 13th, 2021

DOl: https://doi.org/10.21203/rs.3.rs-411401/v1

License: (c) (1) This work is licensed under a Creative Commons Attribution 4.0 International License.

Read Full License 


\section{Abstract}

Objective. To study the correlative between the sesamoid bones under the head of the first metatarsal and the development of hallux valgus determined with radiographs.Methods. The measurements were performed on the X-ray of 300 normal feet and 300 cases of hallux valgus. The following parameters were measured: hallux valgus angle(HVA); the first-second intermetatarsal angle(IMA) between the axes of the first and second metatarsal;the length of the second metatarsal『CD囚; the position of tibial sesamoid(TSP ) measured the percent formed between the tibial sesamoid and the centreline of the first metatarsal,the position of fibular sesamoid(FSP) measured tangent value between fibular sesamoid bone and lateral cortex of first metatarsal bone ; the absolute distances $(A B)$ from the centre of the tibial sesamoid to the long axis of the second metatarsal, the absolute distances (EF) from the centre of the fibular sesamoid to the long axis of the second metatarsal and the absolute distance (GH) from the centre of the tibial sesamoid to the centre of the fibular sesamoid. Then calculate the ratio of $A B$ to $C D$ (K1), EF to CD (K2) and GH to CD (K3). Results.HVA moderately positively correlates with TSP and moderately negatively correlates with FSP in subjects with HVA $\geq 20^{\circ}$. HVA and FSP are strongly negatively correlated in the hallux valgus group. Conclusion.The dislocation of sesamoid bone under the first metatarsal head is an important pathological factor leading to valgus. HVA is positively correlated with TSP and negatively correlated with FSP.

\section{Background}

The sesamoid bones on the first metatarsal head are an important part of the structure and function of the forefoot .It plays an important role in regulating pressure,reducing friction and changing the direction of muscle traction ${ }^{[1-4]}$. Whether the sesamoid bones dislocation is corrected or not is an important index about evaluating the curaive effect of valgus correction ${ }^{[5-8]}$. Many things are puzzling at the moment, there are a few reports about the quantitative index of the normal position of the sesanoid bones on the first metatarsal head and clinical cases about the relationship between sesamoid dislocation and hallux valgus. The image research is blank in the aspects of the sesamoid bones at present. In this study,the correlation between sesamoid bones on the first metatarsal head and hallux valgus was analyzed through the comparative study of normal foot and hallux valgusso, as to provide an important reference for the clinical treatment of hallux valgus and hallux valgus orthosis.

\section{Subjects and Methods}

\subsection{Patients}

In hallux valgus groups (the hallux valgus angle (HVA) $\geq 20^{\circ}$ and the angle formed between the axes of the first and second metatarsal $(\mathrm{IMA})>12^{\circ}$, , measurements were conducted on 300 feet of 300 subjects (42 feet of 42 men, and 258 feet of 258 women; age 14 to 72 years, average $43.4 \pm 2.1$ years); the foot with large HVA was conducted when both feet were hallux valgus. Among them, there were 102 patients with family history $(102 / 300,34.3 \%), 86$ patients with history of trauma $(86 / 300,28.7 \%), 30$ cases of 
shoes with unreasonable design (30/300, $10.0 \%)$, and 82 cases of other patients ( $82 / 300,27.3 \%)$. All patients who visited the hospital were object between 2014 and 2016. On the same period, measurements were conducted on the right foot of 300 subjects in the control group (102 feet of 102 men and 198 feet of 198 women; aged 8 to 74 years, average $43.6 \pm 3.2$ years). Potential subjects were excluded if they had a history of chronic rheumatoid arthritis, cerebral palsy, cerebral infarction, peripheral nerve paralysis, or other disease factors. None of the subjects had a history of operations for treating hallux valgus; patients had previously either received no treatment or had been treated conservatively.

\subsection{Machine and Methods}

\subsubsection{Inspection Method}

The GE Definium 6000 DR filming machine is used to shoot positive and oblique images of the foot. The tube voltage is $60 \sim 65 \mathrm{kV}$, and the tube current is $100 \mathrm{~mA}$. The radiographs of the feet were taken weightbearing with normal and hallux valgus feet to ensure that the foot is perpendicular to the central axis of the tibia and fibula and the rays pass through the back of the foot .

\subsubsection{Measurements}

The following parameters were measured in the X-ray image of the feet with normal and hallux valgus feet: the angle between the first metatarsal axis and the proximal hallux phalanx axis (the hallux valgus angle, HVA); the angle formed (IMA) between the axes of the first and second metatarsal; the length of the second metatarsal (CD); the absolute distances (AB)from the centre of the tibial sesamoid to the long axis of the second metatarsal, the absolute distances (EF) from the centre of the fibular sesamoid to the long axis of the second metatarsal and the absolute distance $(\mathrm{GH})$ from the centre of the tibial sesamoid to the centre of the fibular sesamoid. Relative to the position of the tibial sesamoid to the centreline of the first metatarsal, the percent formed (TSP) between the tibial sesamoid and the centreline of the first metatarsal, and defined by the position of the tibial sesamoid located on the medial of the centreline of the first metatarsal and not to exceed the centreline of the first metatarsal is $0 \%$; the centreline of tibial sesamoid located on the centreline of the first metatarsal axis established is $50 \%$, located on the right of the centreline of the first metatarsal and completely exceeding the centreline is $100 \%$; others are between $0-100 \%$ relative to the position of the fibular sesamoid to the lateral of the first metatarsal. The percent formed (FSP) between the fibular sesamoid and the first metatarsal, and defined by the position of the fibular sesamoid located on the of the lateral of the first metatarsal and not connected to the first metatarsal is $0 \%$. The fibular sesamoid and the first metatarsal completely coincide with $100 \%$ (Figs. 1 and 2). Next, the relative distances, defined as the ratio of these absolute distances to the length of the second metatarsal, were calculated to adjust for foot size. The ratio of $A B$ to $C D$ is $K 1 ; E F$ to $C D$ is $K 2 ; G H$ to $\mathrm{CD}$ is $\mathrm{K} 3$.

\subsection{Statistics}

SPSS 20.0 statistical analysis software was used. First, the measurement data were expressed as \pm s, and two independent sample t-test was used for comparison between the two groups. TSP, FSP, K1, K2 and K3 
are independent variables, HVA and IMA are dependent variables, and multiple linear regression analysis was used in all patient measurements (in the control group and the hallux valgus group) during the study period. We investigated the correlation between the TSP, FSP, K1, K2, K3 and HVA and IMA. The variables with significant differences in the above regression analysis were the dependent variables. Parameter $(P$ $<0.05$ was considered significant) analysis was performed as independent variables, HVA and IMA are dependent variables using the multiple linear regression analysis to investigate the correlation in the control group with $H V A \geq 20$ and in the hallux valgus group (HVA $\geq 20^{\circ}$ and $\left.\mid M A>12\right)$. $|r| \geq 0.8$ was considered strongly correlated; $0.4 \leq|r|<0.8$ was considered moderately correlated; $|r|<0.4$ was considered weakly or not correlated. $\mathrm{P}<0.05$ was considered significant.

\section{Results}

TSP, FSP, K2 and K3 were significantly different in the control group (the normal group) and the hallux valgus group $(P<0.05)$ (able 1), In all subjects, FSP, K2 and K3 are correlated with HVA, and the linear regression equation is HVA = 63.976-10.541FSP-0.446K2-1.115K3; (Table 2) however, only K1 correlates with IMA, and the linear regression equation is IMA $=8.956-0.399 \times \mathrm{K} 1$ (Table 3 ). In hallux valgus group, TSP and FSP were positively correlated with IMA. After correlation using the multiple linear analysis, the variables $\mathrm{K} 2$ and $\mathrm{K} 3$ were excluded. The results show that HVA and TSP are moderately positively correlated, HVA and FSP are moderately negatively correlated in subjects with HVA $\geq 20^{\circ}(r=-0.494, P<$ 0.05 ) (Table4ITThe linear regression equation is HVA $=27.720+5.044 \times \mathrm{TSP}-6.028 \mathrm{FSP} . \beta 95 \% \mathrm{Cl}$ is $(-11.826,-0.231)$; HVA and FSP are strongly negatively correlated in the hallux valgus group $(r=-0.824, P<$ $0.05)$. The linear regression equation is HVA $=26.842+5.021 \times \mathrm{TSP}-6.011 \mathrm{FSP} . \beta 95 \% \mathrm{Cl}$ is $(-12.013$, $-0.141)$ \

Table 1

Comparison of FSP, K2 and K3 between valgus group and control group $( \pm s)$

\begin{tabular}{|lllll|}
\hline & FSP(\%) & TSP $(\%)$ & $\mathrm{K}_{2}$ & $\mathrm{~K}_{3}$ \\
\hline valgus group $(n=300)$ & $26.0 \pm 4.3$ & $56.4 \pm 3.2$ & 0.65 & 1.11 \\
\hline control group $(n=300)$ & $52.3 \pm 3.2$ & $28.2 \pm 2.1$ & 0.46 & 0.82 \\
\hline$t$ & 3.082 & 2.032 & 1.023 & 1.286 \\
\hline
\end{tabular}


Table 2

Multiple linear regression analysis between HVA and FSP, K2, and K3 for the control group and the hallux valgus group

\begin{tabular}{|c|c|c|c|c|c|c|}
\hline & \multicolumn{2}{|c|}{ Coefficient } & \multirow[t]{3}{*}{$\mathbf{t}$} & \multirow[t]{3}{*}{$P$} & \multicolumn{2}{|c|}{$\beta 95.0 \% \mathrm{Cl}$} \\
\hline & $\beta$ & Standard & & & Lower & Upper \\
\hline & & deviations & & & limit & limit \\
\hline (Constant) & 63.976 & 12.322 & 5.192 & 0 & 39.439 & 88.513 \\
\hline FSP & -10.541 & 3.221 & -3.273 & 0.002 & -16.955 & -4.127 \\
\hline K2 & -0.446 & 0.184 & -2.422 & 0.018 & -0.813 & -0.079 \\
\hline K3 & -1.115 & 0.551 & -2.025 & 0.046 & -2.212 & -0.019 \\
\hline
\end{tabular}

Table 3

Correlation analysis between $\mathrm{K} 1$ and IMA in the control group and the hallux valgus group

\begin{tabular}{|c|c|c|c|c|c|c|}
\hline & \multicolumn{2}{|c|}{ Coefficient } & \multirow[t]{2}{*}{$t$} & \multirow[t]{2}{*}{$P$} & \multicolumn{2}{|c|}{$\beta 95.0 \% \mathrm{Cl}$} \\
\hline & $\beta$ & $\begin{array}{l}\text { Standard } \\
\text { deviations }\end{array}$ & & & $\begin{array}{l}\text { Lower } \\
\text { limit }\end{array}$ & $\begin{array}{l}\text { Upper } \\
\text { limit }\end{array}$ \\
\hline (Constant) & 8.956 & 0.304 & 29.445 & 0 & 8.351 & 9.561 \\
\hline K1 & -0.399 & 0.118 & -3.37 & 0.001 & -0.634 & -0.163 \\
\hline
\end{tabular}

Table 4

Correlation analysis between TSP, FSP and HVA in the control group (HVA $\geq 20^{\circ}$ )

\begin{tabular}{|c|c|c|c|c|c|c|c|}
\hline & \multicolumn{2}{|c|}{ Coefficient } & \multirow[t]{2}{*}{$\mathbf{t}$} & \multirow[t]{2}{*}{$P$} & \multicolumn{3}{|c|}{$\beta 95.0 \% \mathrm{Cl}$} \\
\hline & $\beta$ & $\begin{array}{l}\text { Standard } \\
\text { deviations }\end{array}$ & & & $\begin{array}{l}\text { Lower } \\
\text { limit }\end{array}$ & $\begin{array}{l}\text { Upper } \\
\text { limit }\end{array}$ & $|r|$ \\
\hline (Constant) & 27.72 & 1.886 & 14.697 & 0 & 23.946 & 31.494 & \\
\hline TSP & 5.044 & 2.329 & 2.165 & 0.034 & 0.383 & 9.705 & 0.494 \\
\hline FSP & -6.028 & 2.897 & -2.081 & 0.042 & -11.826 & -0.231 & \\
\hline
\end{tabular}

\section{Discussion}




\subsection{The incidence of hallux valgus}

Hallux valgus is a common disease in foot and ankle surgery, which is a kind of foot compound deformity in which the metatarsal toe tilts outward and exceeds the normal physiological angle ${ }^{[9-12]}$. It is characterized by the adduction of the first metatarsal bone, hallux valgus deformity and subluxation of the first metatarsophalangeal joint in severe cases ${ }^{[13]}$. It can be accompanied by other foot deformities such as cross toe, hammer toe, flat foot and so on ${ }^{[14-15]}$. The incidence of hallux valgus was reported in literature as $2 \%-50 \%{ }^{[16]}$ with a completely difference. In 1990 , the National Institutes of Health calculated that the incidence of hallux valgus was $5.1 \%{ }^{[17]}$. There are more females than males, and the ratio of male to female is $1: 4.63^{[18-20]}$. In our study, the average age of hallux valgus patients was $(43.4 \pm 2.1)$ years old, the youngest patient age was 14 years old, and the male to female ratio was 1: 6.14 (42 / 258). At present, the pathogenesis of hallux valgus is not clear, which is closely related to congenital and acquired factors ${ }^{[21-22]}$. The common factors are heredity and trauma ${ }^{[23]}$. Hallux valgus is autosomal dominant hereditary disease ${ }^{[24]}$. In this group, 102 patients (102 / 300) had family genetic history, and the age of onset was generally after 30 years old, while those without genetic history were generally younger, generally before 30 years old. It was reported in previous literature that irrationally designed shoes were the second leading cause of hallux valgus, while trauma ( $86 / 300,28.7 \%)$ was the second leading factor besides heredity in 300 patients with hallux valgus. However, irrationally designed shoes were only found in 30 cases $(30 / 300,10.0 \%)$. Therefore, sesamoid dislocation may be the main factor leading to hallux valgus after trauma.

\subsection{Correlation between sesamoid bones and hallux valgus 3.2.1 Correlation between tibial sesamoid bone and hallux valgus}

It was reported ${ }^{[25]}$ that the following relationships between the sesamoids on the head of the first metatarsal and hallux valgus. The sesamoids on the head of the first metatarsal are in dynamic balance because of pulling by two group muscles. The inside end of the abductor hallucis muscle and the flexor hallucis brevis muscle is the tibial sesamoid. The outside end of the abductor hallucis muscle and the flexor hallucis brevis muscle is the fibular sesamoid. When the dynamic balance of the two groups of muscles is broken, it weakens the strength of the abductor hallucis muscle or increases the strength of the flexor hallucis brevis muscle. All of these factors can cause the occurrence of hallux valgus, and when the HVA exceeds $30 \sim 35^{\circ}$, the abductor hallucis tendon is displaced plantarward, and the adductor hallucis, flexor hallucis brevis, and abductor hallucis rotates in a lateral direction. This usually leads to pronation of the ankle, and the abductor muscles inside the flexion axis of the first metatarsophalangeal joint normally moved towards the ankle. Laterally, the adductor muscles that lost the epicondylar abductor muscles further pull the metatarsal to create eversion, pulling the medial capsular ligament, especially the osseous capsule of the joint capsule, and making the medial metatarsophalangeal joint capsule tense through the "capstan mechanism". The pulling force on the inside of the first metatarsal 
head is generated, and at the same time, the lateral side of the humeral head is pushed and the first metatarsal inversion is aggravated, resulting in relative sesamoid subluxation. The majority of the tibial sesamoid in normal foot are located on the inside of the central longitudinal axis of the first metatarsal shaft ${ }^{[26]}$. Hardy and Clapham ${ }^{[13]}$ reported the seven-position scale for sesamoid displacement from the AP view. They reported that a correlation was found between the stage of the position of the sesamoid and the hallux valgus angles. Yildirim ${ }^{[14]}$ reported the four-position scale for sesamoid displacement from the axis view. Judgement standard was the relationship between the position and the bony crest of the first metatarsal. That is, the tibial sesamoid bone did not exceed the bony crest of the first metatarsal at 0 degrees, less than $50 \%$ is 1 degree, more than $50 \%$ are 2 degrees and completely exceeding the bony crest of the first metatarsal is 3 degrees. This method can explain the subluxation of the sesamoid. The results of Aper RL, Saltzm an CL, B row n TD [10] showed that there were different degrees of sesamoid dislocation in hallux valgus, and the more obvious the dislocation of sesamoid bone was, the larger the HVA and IMA were $(P<0.05)$. There was a significant correlation between sesamoid dislocation and HVA. The correlation number between sesamoid dislocation and HVA was $0.47(P<0.05)$. The correlation coefficient with IMA was $0.43(P<0.05)$. There was no correlation between the distance from the tibial sesamoid bone to the second metatarsal axis and the ratio of the second metatarsal length $K 1(P>0.05)$. In this study, through the measurement and statistics of the normal foot, it was found that there was no significant correlation between HVA and TSP $(P=0.0445)$. IMA was only correlated with K1 $(T=3.37, P=$ 0.001 ) (Table 5). The results are not consistent with the study of Aper RL, Saltzm an CL, B row $n$ TD. Aper $R L$ only measured the $K 1$, while this study measured the $A B, G H, K 1$, and $K 3$. HVA was correlated with $K 1$ and $\mathrm{K} 3(\mathrm{~T}=-3.273 \square \mathrm{P}=0.002$ and $\mathrm{T}=-2.025 \square \mathrm{P}=0.046)$ in the normal foot. The results show that TSP was moderately correlated with HVA and IMA in subjects with HVA $>20^{\circ}(|r|=0.494, \mathrm{P}<0.05)$. TSP, FSP and HVA and IMA are strongly positively correlated in the hallux valgus group $(|r|=0.824, P<0.05)$. In other words, the more intersecting of the central axis of the tibial sesamoid bone and the first metatarsal bone, the larger the valgus angle (HVA). When the HVA $>30^{\circ}$, the tibial sesamoid bone tends to deviate to the lateral side of the central axis of the first metatarsal bone. This is similar to AperRL,SaltzmanCL,BrownTD. Through measurement and statistics of the normal foot group, we found that the normal position of the tibial sesamoid bone (TSP) is $17.8 \%-34.3 \%$ (intersection values between the tibial sesamoid bone on the head of the first metatarsal and the central longitudinal axis of the first metatarsal shaft), with an average of $(28.2 \pm 2.1) \%$. At present, the normal position of TSP has not been reported at home and abroad. The discovery of this quantitative index lays a foundation for future study of sesamoid lesion on the first metatarsal head. 3.22 Correlation between fibular sesamoid bone and hallux valgus When the relationship between the sesamoid bones and the first metatarsal head changes for various reasons, the dynamic fulcrum of the sesamoid bones movement shifts, gradually forming the metatarsal seed joint and causing fatigue and strength changes of the surrounding muscles or tendons (the strength of the lateral muscle group increases and the strength of the medial muscle group weakens) [26-28]. So the metatarsal toe gradually tilts to the outside, which makes a normal line of force change ${ }^{[29]}$. While the metatarsal head tries its best to move medially in order to maintain the support of the foot. When the first metatarsal head is pulled by the toe seed ligament and other soft tissues, the first metatarsal head is pronated, resulting in valgus deformity ${ }^{[30]}$. Another theory holds that the abductor 
muscle and adductor muscle and the flexor brevis muscle form a joint stop, part of which ends on the tibial sesamoid bone. The original balance of the muscle strength of the metatarsal bone promotes the metatarsal rotation during the relative displacement of the sesamoid bone. The sesamoids on the head of the first metatarsal are in dynamic balance because of pulling by two group muscles ${ }^{[31]}$. So in theory, either sesamoid dislocation could lead to hallux valgus ${ }^{[32-33]}$.In this study, it was found that the normal position of sesamoid bone (FSP) intersected with the lateral cortex of the first metatarsal bone accounted for $(52.3 \pm 3.2) \%$ of the total area of sesamoid bones. The purpose of this study was to find a quantitative index for judging the dislocation of sesamoid bones on the first metatarsal head. Among all the subjects in this group, HVA was correlated with FSP, K2 and K3. The linear regression equation was HVA $=63.976-10.541 \mathrm{FSP}-0.446 \mathrm{~K} 2-1.115 \mathrm{~K} 3$. However, the values of K2 and K3 were excluded by multiple linear regression equation analysis, which suggested that there was no obvious linear correlation between the values of $\mathrm{EF}$ and $\mathrm{GH}$ and hallux valgus, while there was a linear relationship between FSP and HVA in the subjects with HVA $\geq 20^{\circ}$. There was a high negative correlation between HVA and FSP in patients with hallux valgus, suggesting that FSP is an important pathological factor of hallux valgus.

Table 5

Correlation analysis between TSP, FSP and HVA in the hallux valgus group

\begin{tabular}{|c|c|c|c|c|c|c|c|}
\hline & \multicolumn{2}{|c|}{ Coefficient } & \multirow[t]{2}{*}{$t$} & \multirow[t]{2}{*}{$P$} & \multicolumn{2}{|c|}{$\beta 95.0 \% \mathrm{Cl}$} & \multirow[t]{2}{*}{$|\mathbf{r}|$} \\
\hline & $\beta$ & $\begin{array}{l}\text { Standard } \\
\text { deviations }\end{array}$ & & & $\begin{array}{l}\text { Lower } \\
\text { limit }\end{array}$ & $\begin{array}{l}\text { Upper } \\
\text { limit }\end{array}$ & \\
\hline (Constant) & 26.842 & 1.623 & 15.584 & 0 & 24.186 & 30.925 & \\
\hline TSP & 5.021 & 2.314 & 4.901 & 0.027 & 0.376 & 9.842 & 0.824 \\
\hline FSP & -6.011 & 2.865 & -5.081 & 0.018 & -12.013 & -0.141 & \\
\hline
\end{tabular}

\subsection{The deficiency of this study}

Improper body position and measurement errors may be the main factors affecting the results of this study. At present, there is no unified standard for the measurement of TSP and FSP. In this study, visual method is used to determine TSP and FSP. This method is greatly affected by subjective factors, which can also affect the results of this study.

In short, We believe that our study is significant for the treatment and evaluation of hallux valgus, especially given that the relationship between fibular sesamoid bone and hallux valgus has not been reported; therefore, through the results of our study, subluxation of the tibial sesamoid and fibular sesamoid should be corrected at the same time in clinical correction surgery for hallux valgus.

\section{Abbreviations}


HVAØhallux valgus angle

IMA囚the first-second intermetatarsal angle

TSP 『the position of tibial sesamoid

FSP囚the position of fibular sesamoid

\section{Declarations}

\section{Ethics approval and consent to participate}

Approval from the Institutional Review Board was obtained and in keeping with the policies for a retrospective review, informed consent was not required."

Consent for publicationNot applicableAvailability of data and materialData sharing not applicable to this article as no data-sets were generated or analyzed during the current study. If you do not wish to publicly share your data, please write: "Please contact author for data requests." Competing interestsThe authors declare that they have no competing interests. FundingNot applicableAuthors' contributionsXiaozhong Li carried out the measurements studies, drafted the manuscript. Dongxue Liu participated in the design of the study and performed the statistical analysis. Xufang Wang conceived of the study, and participated in its design and coordination and helped to draft the manuscript. All authors read and approved the final manuscript. Corresponding authorCorrespondence to Xiaozhong Li. Acknowledgements Not applicableAuthors' information Xiaozhong Li

Department of Radiology囚University Hospital of Gansu Traditional Chinese Medicine

\section{Dongxue Liu}

University Hospital of Gansu Traditional Chinese Medicine

\section{Xufang Wang}

University Hospital of Gansu Traditional Chinese Medicine

\section{References}

[1]Hillier J. C., Peace K., Hulme A. \& Healy J. C. (2004). Pictorial review: MRI features of foot and ankle injuries in ballet dancers. TheBritish journal of radiology,77(918),532-537. https://doi.org/10.1259/bjr/60447506.

[2]Karasick D.,\&Wapner K.L.(1990).Hallux valgus deformity: preoperative radiologic assessment.AJR.Americanjournal of roentgenology,155(1),119-123.

https://doi.org/10.2214/ajr.155.1.2112832. 
[3]Hwang SH, Lee SC, Nam CH, et al. (2017).The treatment for hallux valgus with Scarf osteotomy in elderly patients with osteoporosis. Journal of Korean Foot and Ankle Society ,21(3),93-97. doi:10.14193/jkfas.2017.21.3.93.

[4]Rakesh Dalal,Rajesh Rachha,David Leonard,Ajay Chourasia,Saqib Javed.(2017).Short Scarf Osteotomy for Hallux Valgus: Short-term and Medium-term Results - Original Article.Journal of Foot and Ankle Surgery (Asia Pacific),Vol.4(No.1),14-18.doi:10.5005/jp-journals-10040-1063.

[5]Richardson E. G. (1999). Hallucal sesamoid pain: causes and surgical treatment. The Journal of the American Academy of Orthopaedic Surgeons, 7(4), 270-278. https://doi.org/10.5435/00124635$199907000-00007$.

[6]Mckeever D. C. (1952). Arthrodesis of the first metatarsophalangeal joint for hallux valgus, hallux rigidus, and metatarsus primus varus. The Journal of bone and joint surgery. American volume, 34-A(1), 129-134.

[7]Patil S.D., Patil V.D., Khan A.\& Khanore C. (2016). Correction of a Forefoot Deformity Caused by a Large, Solitary Metatarsal Osteochondroma in an Adolescent: A Case Report. The Journal of foot and ankle surgery: official publication of the American College of Foot and Ankle Surgeons,55(2), 427-433. https://doi.org/10.1053/j.jfas.2015.02.015.

[8]Schein A. J., Skalski M. R., Patel D. B., et al.(2015). Turf toe and sesamoiditis: what the radiologist needs to know. Clinical imaging, 39(3), 380-389. https://doi.org/10.1016/j.clinimag.2014.11.011.

[9]Cohen B.E. (2009). Hallux sesamoid disorders. Foot and ankle clinics, 14(1),91-104. https://doi.org/10.1016/j.fcl.2008.11.003.

[10]Aper R. L., Saltzman C. L., \& Brown T. D. (1996). The effect of hallux sesamoid excision on the flexor hallucis longus moment arm. Clinical orthopaedics and related research, (325), 209-217. https://doi.org/10.1097/00003086-199604000-00025.

[11]Bartosiak K., \& McCormick J. J. (2019). Avascular Necrosis of the Sesamoids. Foot and ankle clinics, 24(1), 57-67. https://doi.org/10.1016/j.fcl.2018.09.004.

[12]Richardson E. G. (1999). Hallucal sesamoid pain: causes and surgical treatment. The Journal of the American Academy of Orthopaedic Surgeons,7(4),270-278. https://doi.org/10.5435/00124635$199907000-00007$.

[13]Hardy R.H.\& CLAPHAM J. C. (1951). Observations on hallux valgus; based on a controlled series. The Journal of bone and joint surgery. British volume, 33-B(3), 376-391. https://doi.org/10.1302/0301620X.33B3.376.

[14]Yildirim R,Anjum SN,Nichol JE.(2011).Sesamoids of the foot.Orthopaedics Current ,19(8), 40-48. 
[15]Fatih Dogar.(2016).Distal Metatarsal Osteotomy in Hallux Valgus Surgery: Chevron Osteotomy.Journal of Clinical and Analytical Medicine,Vol.6(No.157),752-755.doi:10.4328/jcam.3553.

[16] Ohmori T., Katsuo S., Sunayama C., et al. (2016). Irreducible plantar dislocation of the interphalangeal joint of the great toe due to an accessory sesamoid bone: a case report. Archives of orthopaedic and trauma surgery, 136(4),533-537. https://doi.org/10.1007/s00402-015-2379-3.

[17]Alfaraj D. N.,Vilke G. M. (2017). Tripartite Fracture of the Ulnar Sesamoid Bone of the Thumb. The Journal of emergency medicine,53(5),758-759. https://doi.org/10.1016/j.jemermed.2017.08.020.

[18] Hood C. R., Jr Miller J. R., Eldridge S., \& Tran M. (2017). The Rare Lesser Metatarsal Bipartite Sesamoid A Case Report. Journal of the American Podiatric Medical Association, 107(6), 548-550. https://doi.org/10.7547/16-114.

[19]Schein A. J., Skalski M. R., Patel D. B.,et al. (2015). Turf toe and sesamoiditis: what the radiologist needs to know. Clinical imaging,39(3),380-389. https://doi.org/10.1016/j.clinimag.2014.11.011.

[20]Bartosiak K., \& McCormick J. J. (2019). Avascular Necrosis of the Sesamoids. Foot and ankle clinics, 24(1), 57-67. https://doi.org/10.1016/j.fcl.2018.09.004.

[21]Wakely C D, Johnson CP, Watt I.(2007). The value of MR imaging in the diagnoses of the os trigonum syndrome. Skeletal Radiol, 25(2):133-136.doi:10.1007/s002560050049.

[22]Howse A. J. (1982). Posterior block of the ankle joint in dancers. Foot \& ankle, 3(2), 81-84. https://doi.org/10.1177/107110078200300205.

[23]lovane A., Midiri M., Finazzo M., Carcione A., De Maria M., \& Lagalla R. (2000). Sindrome dell'os trigonum tarsi. Ruolo della Risonanza Magnetica [Os trigonum tarsi syndrome. Role of magnetic resonance]. La Radiologia medica, 99(1-2), 36-40.

[24]Hillier J. C., Peace K., Hulme A.\& Healy J. C. (2004). Pictorial review: MRI features of foot and ankle injuries in ballet dancers. The British journal of radiology, 77(918), 532-537. https://doi.org/10.1259/bjr/60447506.

[25]Lee J. C., Calder J. D.\& Healy J. C. (2008). Posterior impingement syndromes of the ankle. Seminars in musculoskeletal radiology,12(2),154-169. https://doi.org/10.1055/s-2008-1078703.

[26]Robinson P., \& White L. M. (2002). Soft-tissue and osseous impingement syndromes of the ankle: role of imaging in diagnosis and management. Radiographics : a review publication of the Radiological Society of North America, Inc,22(6),1457-1471. https://doi.org/10.1148/rg.226025034.

[27]Linklater J. (2009). MR imaging of ankle impingement lesions. Magnetic resonance imaging clinics of North America, 17(4), 775. https://doi.org/10.1016/j.mric.2009.06.006. 
[28]Grace D. L. (2000). Sesamoid problems. Foot and ankle clinics, 5(3), 609-627.

[29]Stroh K. I., Altman M. I., \& Yee D. Y. (1990). First metatarsophalangeal joint arthrodesis. Treatment for sesamoid fractures. Journal of the American Podiatric Medical Association, 80(11), 595-599. https://doi.org/10.7547/87507315-80-11-595.

[30]Richardson E. G. (1999). Hallucal sesamoid pain: causes and surgical treatment. The Journal of the American Academy of Orthopaedic Surgeons,7(4),270-278. https://doi.org/10.5435/00124635$199907000-00007$.

[31] Heineman N., Liu G., Pacicco T., Dessouky R., Wukich D. K.\& Chhabra A. (2020). Clinical and imaging assessment and treatment of hallux valgus. Acta radiologica (Stockholm, Sweden : 1987), 61(1), 56-66. https://doi.org/10.1177/0284185119847675.

[32]Filippi J., \& Briceno J. (2020). Complications after Metatarsal Osteotomies for Hallux Valgus: Malunion Nonunion Avascular Necrosis and Metatarsophalangeal Osteoarthritis. Foot and ankle clinics,25(1),169-182. https://doi.org/10.1016/j.fcl.2019.10.008.

[33]Cavalheiro C. S., Arcuri M. H., Guil V. R.\& Gali J. C. (2020). Hallux Valgus Anatomical Alterations And Its Correlation With The Radiographic Findings. Acta ortopedicabrasileira,28(1),12-15. https://doi.org/10.1590/1413-785220202801226897.

\section{Figures}

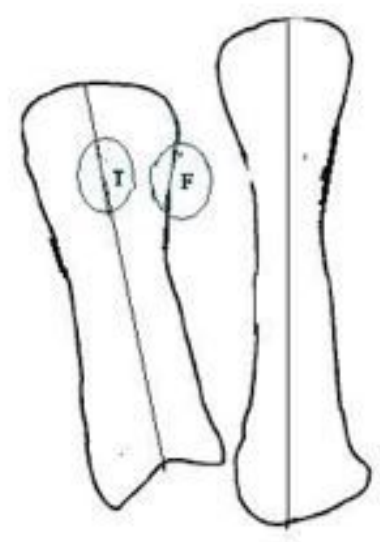

\section{Figure 1}

the position of the tibial sesamoid is $50 \%$, the position of the fibular sesamoid is $50 \%$. 


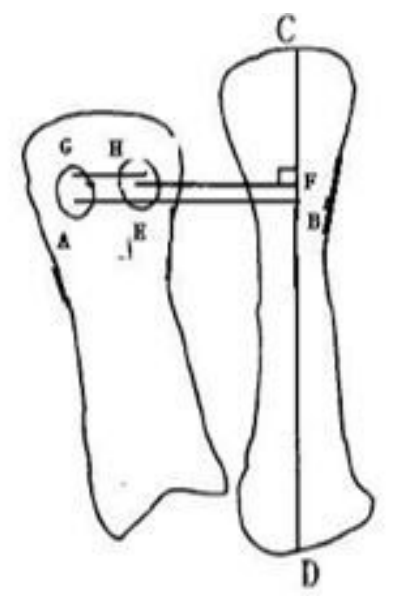

Figure 2

$A$ and $\mathrm{G}$ are the centre of the tibial sesamoid, $\mathrm{E}$ and $\mathrm{H}$ are the centre of the fibular sesamoid. The length of the second metatarsal (CD); the absolute distances from the centre of the tibial sesamoid to the long axis of the second metatarsal $(A B)$,the absolute distances from the centre of the fibular sesamoid to the long axis of the second metatarsal $(\mathrm{EF})$ and the absolute distance from the centre of the tibial sesamoid to the centre of the fibular sesamoid (GH).

\section{Supplementary Files}

This is a list of supplementary files associated with this preprint. Click to download.

- Listofabbreviations.doc 